\title{
Investigação experimental da utilidade da medicação no pós-operatório em cirurgia refrativa
}

\section{Experimental investigation of postoperative useof medication in refractive surgery}

\author{
Sâmia Ali Wahab ${ }^{1}$ \\ Hamilton Moreira ${ }^{2}$ \\ Michele Buquera $^{3}$ \\ Luciane Moreira ${ }^{4}$ \\ Alessandra Castelano Daros ${ }^{5}$ \\ Cínara Sakuma de Oliveira ${ }^{6}$
}

Trabalho realizado no Hospital Universitário Evangélico de Curitiba.

${ }^{1}$ Mestre em princípios da cirurgia pelo Instituto de Pesquisas Médicas - IPEM.

2 Doutor em oftalmologia pela Universidade Federal de São Paulo - UNIFESP. São Paulo (SP); Professor adjunto da Universidade Federal do Paraná. Professor Assistente da Faculdade Evangélica do Paraná.

${ }^{3}$ Médica do corpo clínico do Hospital de Olhos do Paraná.

${ }^{4}$ Mestre em princípios da cirurgia pelo Instituto de Pesquisas Médicas - IPEM.

5 Médica do corpo clínico do Hospital de Olhos do Paraná.

${ }^{6}$ Mestre em princípios da cirurgia pelo Instituto de Pesquisas Médicas - IPEM.

Endereço para correspondência: Sâmia Ali Wahab. Al. Princesa Isabel, 1464 - Apto. 22 - Curitiba (PR) CEP 80730-080 - E-mail: saliw@terra.com.br

Recebido para publicação em 11.07.2003

Versão revisada recebida em 26.11.2004

Aprovação em 15.12.2004

Nota Editorial: Após concluída a análise do artigo sob sigilo editorial e com a anuência do Dr. Eduardo Melani Rocha sobre a divulgação de seu nome como revisor dele, agradecemos sua participação nesse processo.

\begin{tabular}{l} 
RESUMO \\
\hline Objetivo: Isolar microrganismoem microceratótomo, induzir ceratite infec- \\
ciosa avaliando a resposta terapêutica. Métodos: Foram realizadas coletas \\
consecutivas de material a partir do microceratótomo usado rotineiramente \\
para cirurgia refrativa em olhos humanos durante um período de 8 dias. \\
Preparou-se uma diluição segundo a escala de MacFarland para inocular \\
na córnea de cobaias. Utilizaram-se oito animais, injetou-se $10 \mu$ no \\
estroma corneano de ambos os olhos. No olho direito não se instilou \\
medicamento (grupo 1 ) e no olho esquerdo instilou-se ciprofloxacina $0,3 \%$ \\
associado a dexametasona a 0,1\% e trometamina de cetorolac de 4 em 4 \\
horas por 24 horas (grupo 2). Após este período as cobaias foram \\
sacrificadas e as córneas divididas, sendo uma metade enviada para \\
análise microbiológica e outra para histopatologia. Resultados: No grupo \\
sem tratamento, todas as 8 amostras foram positivas, ao passo que no \\
grupo tratado, 3 desenvolveram cultura positiva para Staphylococcus \\
aureus (p=0,0128). A avaliação histopatológica de polimorfonucleares \\
e monomorfonucleares no estroma mostrou que eram menos numerosos \\
no grupo tratado (grupo 2), com p=0,0203 e p=0,0051, respectivamente. \\
Conclusão: Este estudo portanto sugere que o uso de antibiótico de amplo \\
espectro associado a antiinflamatório tem efeito significativo na diminui- \\
ção do processo inflamatório e infeccioso em possível contaminação intra- \\
estromal após cirurgia refrativa.
\end{tabular}

Descritores: Ceratite/microbiologia; Ceratite/quimioterapia; Controle de infecções; Staphylococcus aureus/isolamento \& purificação; Estudos de avaliação; Animal

\section{INTRODUÇ̃̃̃O}

A cirurgia para correção de ametropias e cirurgia da catarata está entre os procedimentos cirúrgicos mais realizados pelos oftalmologistas na atualidade. Considerando o grande número de cirurgias realizadas, são procedimentos com baixa incidência de complicações ${ }^{(1)}$.

A infecção merece destaque pela potencial morbidade que possui, entre todos os riscos que envolvem as cirurgias refrativas ${ }^{(2)}$. Embora as cirurgias refrativas com excimer laser, entre elas o "laser assisted in situ keratomileusis" (LASIK), sejam consideradas menos suscetíveis a infecções, se compararmos com a ceratotomia radial $(\mathrm{RK})^{(3)}$, esta complicação ainda assombra os cirurgiões.

Em muitos estudos, a infecção não é citada em suas casuísticas ${ }^{(4-11)}$. Outros citam apenas casos esporádicos de infecção em pós-operatório de cirurgia refrativa ${ }^{(12-13)}$, demonstrando assim que, apesar de a infecção ser uma complicação temida, não é comum. 
É crítico o fato de que, na cirurgia refrativa, os olhos tratados são olhos saudáveis, pois os pacientes apresentam uma grande expectativa sobre o resultado cirúrgico ${ }^{(3)}$.

Cientes da preocupação de estarmos manipulando olhos sadios na cirurgia refrativa, todos os estudos são realizados para minimizar qualquer eventual complicação per ou pósoperatória que possa ocorrer.

\section{OBJETIVO}

Isolar microrganismo proveniente do instrumental utilizado em cirurgia refrativa, na técnica LASIK.

Induzir ceratite infecciosa experimental com o microrganismo oriundo do primeiro passo.

Avaliar a resposta terapêutica ao uso de medicação utilizada no pós-operatório de cirurgia refrativa.

\section{MÉTODOS}

Para obter o microrganismo, realizou-se coleta de material do microceratótomo com zaragatoa umedecida em BHI (Brain heart infusion), no término da cirurgia (LASIK), na própria mesa cirúrgica, sem qualquer outra manipulação, durante oito dias consecutivos e colocado o material coletado em meio de enriquecimento (BHI). O procedimento cirúrgico foi realizado por profissionais diferentes. Realizou-se também coleta de material do microceratótomo logo após a sua esterilização em autoclave. As amostras obtidas foram enviadas para análise laboratorial, que consistia de cultura, identificação de microrganismos e teste de sensibilidade antimicrobiana à antibioticoterapia proposta. Todas as amostras colhidas para cultura foram semeadas em meio sólido (ágar sangue) e líquido (BHI). Considerou-se positivo o crescimento de microrganismos em qualquer um dos meios inoculados.

O microrganismo selecionado para inoculação e indução de ceratite infecciosa foi aquele que isolamos o maior número de vezes na cultura. Após essa identificação o microrganismo foi preparado para inoculação.

Sob condições estéreis, a colônia bacteriana com maior vitalidade foi isolada no laboratório de microbiologia e diluída em tubo de ensaio com cinco mililitros de solução salina isotônica. Foram realizadas várias diluições, segundo a escala de MacFarland, sendo a solução 0,5 utilizada para a inoculação das cobaias. A diluição 0,5 na escala de MacFarland corresponde aproximadamente a $10^{8} \mathrm{de}$ unidades formadoras de colônia (UFC) por mililitros.

Sob anestesia geral, foram utilizadas oito cobaias-machos, pesando em média $600 \mathrm{~g}$, para inoculação de microrganismos no estroma corneano das mesmas.

A medicação utilizada para anestesia foi xilasina $2 \% 5$ a $10 \mathrm{mg} / \mathrm{kg}$ e quetamina 50 a $80 \mathrm{mg} / \mathrm{kg}$ que apresenta uma duração média de 2 horas.

Sob microscópio cirúrgico, utilizando uma seringa de insulina agulhada, foi realizada injeção corneana intra-estromal, de
$10 \mu l$ da diluição preparada, em ambos os olhos dos animais. A agulha foi introduzida pela periferia e visando o estroma corneano. A solução foi injetada na porção central. As injeções foram realizadas pelo mesmo cirurgião visando à mesma profundidade, a metade anterior do estroma na região central dos dois olhos das cobaias. A agulha foi retirada lentamente, observando-se a formação de uma área esbranquiçada na área central corneana.

O olho direito foi mantido sem medicação. No olho esquerdo, instilou-se a associação de ciprofloxacina $0,3 \%$ com dexametasona $0,1 \%$ e cetorolac de trometamina tópicos, de $4 \mathrm{em} 4$ horas, durante o período de 24 horas. Os colírios foram instilados nesta ordem, primeiro a ciprofloxacina $0,3 \%$ associada à dexametasona $0,1 \%$ e depois o cetorolac de trometamina.

As cobaias foram sacrificadas com injeção intracardíaca de xilasina $2 \%$, sob anestesia geral em técnica previamente descrita e foram retiradas as suas córneas. Cada córnea foi seccionada ao meio no sentido longitudinal, sendo uma metade colocada em formalina $10 \%$ e enviada para análise histopatológica, a outra metade colocada em meio líquido de BHI e enviada para análise laboratorial.

Para análise histopatológica os espécimens foram acondicionados em frascos contendo aproximadamente $5 \mathrm{ml}$ de formalina $10 \%$. Após o tempo necessário para fixação do material, seguindo os critérios laboratoriais de patologia, as córneas foram preparadas para inclusão em blocos de parafina, e, posteriormente, levadas ao micrótomo para preparação das lâminas. Os cortes foram corados com hematoxilina/eosina e examinados em microscopia óptica com aumento de 10 e 40 vezes. Todas as amostras foram mascaradas e examinadas pelo mesmo patologista.

As análises histopatológicas foram feitas de acordo com a camada corneana: epitélio, estroma e endotélio.

Foram avaliados os infiltrados de células polimorfonucleares $(\mathrm{PMN})$ e monomorfonucleares (MMN), sendo classificados em discretos (até 5 células por campo de grande aumento - CGA), moderados (entre 6 e 20 células por CGA) e severos (mais de 20 células por CGA), no estroma.

A presença de colônia bacteriana foi avaliada no epitélio, endotélio e estroma.

Para análise laboratorial, o material que estava no meio de enriquecimento (BHI) foi semeado também em ágar sangue.

Considerou-se positivo o crescimento de microrganismos em qualquer um dos meios inoculados. Quando o microrganismo identificado no meio sólido e líquido foi diferente, considerou-se o resultado positivo para cada amostra e as duas cepas foram identificadas e submetidas aos testes de suscetibilidade antimicrobiana.

Para análise estatística, recorreu-se à análise descritiva dos dados através de tabelas e gráficos. Para a comprovação dos objetivos levantados neste estudo foi utilizado o teste não-paramétrico "Exato de Fisher" (através do "software Epiinfo"). O nível de significância (probabilidade de significância) máximo adotado foi de $5 \%(0,05)$.

O presente estudo foi aprovado pelo Comitê de Ética em Pesquisa da Sociedade Evangélica Beneficente de Curitiba. 


\begin{tabular}{|l|cccc|}
\hline \multicolumn{4}{|c|}{ Tabela 1. Avaliação geral dos dados em estudo } \\
Histopatologia & $\begin{array}{c}\text { Grupo } \\
(\mathbf{n = 8})\end{array}$ & $\begin{array}{c}\text { Grupo 2 } \\
(\mathbf{n = 8})\end{array}$ & Estatística & \\
1. Colônia & & & & \\
- Epitélio & 4 & - & $\mathrm{p}=0,0385$ & $\mathrm{~S}$ \\
- Estroma & 3 & - & $\mathrm{p}=0,1000$ & $\mathrm{NS}$ \\
- Endotélio & 4 & 3 & $\mathrm{p}=0,5000$ & $\mathrm{NS}$ \\
2. Estroma & & & & \\
- Polimorfonuclear & & & $\mathrm{p}=0,0203$ & $\mathrm{~S}$ \\
$\quad$ - Discreto & 1 & 6 & & \\
$\quad$ - Moderado & 4 & 2 & & \\
$\quad$ - Acentuado & 3 & - & & \\
- Monomorfonuclear & & & $\mathrm{p}=0,0051$ & $\mathrm{~S}$ \\
$\quad$ - Discreto & 1 & 7 & & \\
$\quad$ - Moderado & 3 & 1 & & \\
$\quad$ - Acentuado & 4 & - & & \\
3. Cultura & & & $\mathrm{p}=0,0128$ & $\mathrm{~S}$ \\
- Negativa \\
- Positiva
\end{tabular}

\section{RESULTADOS}

Das nove amostras colhidas do microceratótomo enviadas para cultura obtivemos: três amostras negativas, desenvolvimento de Staphylococcus aureus em quatro amostras, desenvolvimento de Staphylococcus epidermidis em duas amostras e a amostra proveniente da autoclave foi negativa.

O número de unidades formadoras de colônias (UFC) isoladas do Staphilococcus aureus foram 6 e 8 e de Staphylococcus epidermidis 5.

$\mathrm{Na}$ avaliação clínica, após vinte e quatro horas da inoculação intra-estromal de $10 \mu \mathrm{l}$ da diluição, todas as córneas apresentaram infiltrado estromal arredondado, central desenvolvendo clinicamente ceratite infecciosa.

$\mathrm{Na}$ avaliação histopatológica do epitélio, houve uma diferença estatisticamente significativa $(\mathrm{p}=0,0385)$, pois houve presença de colônia em 4 amostras do grupo 1 e nenhuma no grupo 2. Entretanto não foi observada diferença significativa na avaliação histopatológica do estroma $(\mathrm{p}=0,1)$ apesar de serem observadas colônias bacterianas no grupo não medicado (grupo 1).

$\mathrm{Na}$ avaliação histopatológica do estroma, em relação aos polimorfonucleares foi observado que o grau mais intenso do processo inflamatório, moderado (50\%) e acentuado (37,5\%), estava mais freqüentemente presente no grupo não medicado $(87,5 \%)(p=0,0203)$, quando comparado com o grupo medicado que apresentava processo inflamatório discreto $(75 \%)$ e moderado $(25 \%)$.

$\mathrm{Na}$ avaliação do estroma também foi observada a presença de monomorfonucleares com maior intensidade, moderado $(37,5 \%)$ e acentuado $(50 \%)$, no grupo $1(87,75 \%)(\mathrm{p}=0,0051)$, comparado com o grupo medicado (grupo 2) que apresentou discreto $(87,5 \%)$ e moderado $(12,5 \%)$.
No endotélio foi observada colônia bacteriana nos dois grupos sem diferença significante $(\mathrm{p}=0,5)$.

A cultura foi positiva para todas as amostras do grupo não tratado $(n=8)$, destas 5 amostras a cultura foi negativa $(62,5 \%)$ e em 3 positivas, com desenvolvimento de Staphylococcus aureus na cultura, $(37,5 \%)(\mathrm{p}=0,0128)$.

\section{DISCUSSÃO}

O material coletado do microceratótomo foi semeado em ágar sangue, pois este é um meio de crescimento geral e, sendo um meio sólido, conseguimos diferenciar mais facilmente o crescimento de colônias de microrganismos diferentes. No meio líquido há apenas a turvação do meio quando inicia um crescimento de microrganismo. Estudo realizado com o objetivo de avaliar as alterações da microbiota conjuntival e palpebral após o uso de antibióticos em cirurgia de catarata e refrativa, os meios de cultura para a avaliação laboratorial foram ágar sangue e $\mathrm{BHI}^{(14)}$.

A cepa de Staphylococcus aureus utilizada neste estudo foi oriunda de cultura de material de microceratótomo. Com isso, tentamos simular uma contaminação per operatória com desenvolvimento de ceratite infecciosa e verificar a evolução em vinte e quatro horas, utilizando apenas a medicação que se usa como profilática de rotina como forma de tratamento da ceratite infecciosa induzida em cobaia.

A endoftalmite infecciosa exógena no pós-operatório de cirurgias oftalmológicas tem como principal agente etiológico Staphylococcus sp tanto nas séries do Brasil quanto nos de outros países ${ }^{(15-16)}$. Geralmente esses microrganismos são encontrados na margem palpebral e no saco conjuntival tendo acesso à câmara anterior ${ }^{(17)}$. Estes estudos corroboram com o agente isolado na cultura do microceratótomo.

A escolha destas medicações, ciprofoloxacina $0,3 \%$ associada à dexametasona $0,1 \%$ e trometamina de cetorolac, para $\mathrm{o}$ grupo medicado (grupo 2) deve-se ao fato de ser sugerido o uso destas classes de drogas tópicas de rotina para o pósoperatório da cirurgia refrativa ${ }^{(2,18-20)}$, sendo a intenção do estudo verificar a ação dessas drogas usadas no pós-operatório numa eventual ceratite infecciosa.

É freqüente o uso de lentes de contato com função protetora e de curativo no pós-operatório imediato, tanto no LASIK quanto no PRK ${ }^{(21)}$. A colonização das lentes de contato gelatinosas usadas no pós-operatório de cirurgias refrativas por bactérias podendo ter um potencial risco de infecção, pela contaminação sugerindo a sua profilaxia foi demonstrada em estudo $^{(22)}$.

Foi utilizada a diluição 0,5 da escala de Macfarland para a injeção intra-estromal, pois esta é a padronização da NCCLS (National Committee for Clinical Laboratory Standarts) para realização de antibiograma. Neste estudo, o número de unidades formadoras de colônia (UFC) provenientes do microceratótomo foram de 5 a 6 UFC, o que demonstra um inóculo baixo.

$\mathrm{O}$ uso de cobaias foi baseado em outros estudos experi- 
mentais sobre infecções corneanas e reação inflamatória ${ }^{(23-27)}$. Este é um animal muito utilizado para testes com vacinas, quando vamos ter um processo infeccioso, dependendo da vacina com uma resposta imunológica.

Foi aplicada injeção intra-estromal, devido à cirurgia refrativa confeccionar uma lamela superficial corneana, podendo ser inoculado algum microrganismo no estroma. Sendo assim, se fosse inoculada alguma bactéria durante a cirurgia, seria provavelmente na interface dessa lamela, e assim, por ocorrer quebra da barreira epitelial e por ter como vetor para inoculação o instrumental cirúrgico, possibilitaria uma infecção, como sugerem alguns estudos, que a infecção seja no leito estromal ${ }^{(28)}$.

Analisando os resultados obtidos da cultura das córneas, verificamos que o antibiótico utilizado inibiu o desenvolvimento do microrganismo. A diluição de 0,5 da escala de Macfarland corresponde aproximadamente a $10^{8}$ (UFC), a que foi inoculada no estroma corneano foi muito superior ao número de UFC isolada no microceratótomo. No grupo não medicado, todas as culturas foram positivas, enquanto no grupo 2 cinco amostras foram negativas e três positivas, mostrando que houve diferença significante ( $\mathrm{p}=0,0128)$.

A ceratite infecciosa foi desenvolvida em todas as córneas, com a intenção de verificar se a medicação utilizada como profilática em cirurgia refrativa teria alguma ação terapêutica em ceratite experimental. Com isso, por analogia, o estudo sugere que essa medicação tem efeito profilático, já que, em uma ceratite infecciosa experimental, no grupo sem medicação a reação inflamatória e infecciosa foi significantemente maior. O tratamento foi apenas para analisar a resposta terapêutica em uma ceratite infecciosa nas primeiras 24 horas de uma medicação utilizada como profilática. As três drogas foram utilizadas para verificar a ação das mesmas no caso de uma infecção.

Na histopatologia, em relação à inflamação estromal, quando inoculamos o agente infeccioso, mostram grande reação inflamatória tanto com polimorfonucleares quanto com monomorfonucleares significantemente maior no grupo não medicado. Com isso, sugere também que, além da atuação do antibiótico sobre o agente infeccioso, temos a ação de antiinflamatório diminuindo os danos teciduais devido à quimiotaxia de polimorfonucleares, como mostram alguns estudos ${ }^{(26,29)}$.

Com a utilização de antibiótico adequado, a piora da ceratite com corticóide foi prevenida ${ }^{(30)}$. Em relato de caso de infecção bilateral pós-LASIK foi por Staphylococcus aureus e as cepas eram sensíveis à fluoroquinolona e a apresentação era no leito da lamela superficial ${ }^{(28)}$.

Apesar da infecção ser uma complicação rara em cirurgia refrativa, devemos tê-la em mente e saber que a medicação utilizada no pós-operatório tem função importante.

\section{CONCLUSÕES}

Foi possível isolar Staphylococcus aureus como microrganismo mais freqüente do microceratótomo após LASIK. Ceratite infecciosa experimental foi induzida, com sucesso, por inoculação intra-estromal do microrganismo isolado proveniente do primeiro passo. A medicação utilizada de rotina no pós-operatório de LASIK, ciprofloxacina $0,3 \%$ associada à dexametasona $0,1 \%$ e trometamina de cetorolac inibiu o crescimento de Staphylococcus aureus. A reação inflamatória foi diminuída com a medicação utilizada neste estudo. A medicação utilizada no pós-operatório de cirurgia refrativa deste estudo tem ação biocida coerente ao microrganismo mais comumente isolado, sugerindo que, com isso diminui o risco de infecção e inflamação associadas.

\section{ABSTRACT}

Purpose: To harvest microorganisms from the microkeratome after LASIK. To induce experimental infectious keratitis and investigate the use of routine postoperative medication. Methods: During eight consecutive days, we cultured the plate of the microkeratome after routine LASIK. Dilutions of the most frequent microorganism was prepared according to the MacFarland scale. Ten microliters were injected into both corneas of eight guinea pigs. The right cornea was used as control and the left cornea was treated with $0.3 \%$ ciprofloxacin associated with dexamethasone and ketorolac tromethamine every 4 hours for 24 hours (group 2). The animals were sacrificed and the corneas were divided into two halves, which were sent to analyzed by the microbiology and histopathology laboratory. Results: In the control group, Staphylococcus aureus could be retrieved from all corneas, while in group 2, only $50 \%$ showed positive cultures $(\mathrm{p}=0.0128)$. Histopathology concerning polymorphonuclear cells showed an intense degree of inflammation in group $1(\mathrm{p}=0.0203)$ and regarding monomorphonuclear cells, it was also more intense in group $1(\mathrm{p}=0.0051)$. Conclusion: This study suggests that the use of wide-spectrum antibiotic associated with anti-inflammatory eye drops has a significant effect on the reduction of a potential infectious and inflammatory process after refractive surgery.

Keywords: Keratitis/microbiology; Keratitis/drug therapy; Infection control; Staphylococcus aureus/isolation \& purification; Evaluation studies; Animal

\section{REFERÊNCIAS}

1. Kanellopoulus AJ, Dreyer EB. Postoperative infection following current cataract extraction surgery. Int Ophthalmol Clin. 1996;36(3):97-107. Review.

2. Kirk R. Basic and clinical science course: External disease and cornea. Section 8. San Francisco: American Academy of Ophthalmology; 2000.

3. Gloor B, Rol P, Fankhauser F, Hoppeler T, Vrbka E. Critical thoughts on current laser surgery of the cornea. Ophthalmologe. 1995;92(4):389-96. Review. Id: German.

4. Davidorf JM, Zaldivar R, Oscherow S. Results and complications of laser in situ keratomileusis by experienced surgeons. J Refract Surg. 1998;14(2):114-22.

5. Buratto L, Ferrari M, Rama P. Excimer laser intrastromal keratomileusis. Am J Ophthalmol. 1992;113(3):291-5.

6. Pesando PM, Ghiringhello MP, Tagliavacche P. Excimer laser in situ keratomileusis for myopia. J Refract Surg. 1997;13(6):521-7.

7. Salz JJ, Maguen E, Macy JI, Papaioannou T, Hofbauer J, Nesburn AB. One- 
year results of excimer laser photorefractive keratectomy for myopia. Refract Corneal Surg. 1992;8(4):269-73.

8. Dulaney DD, Barnet RW, Perkins SA, Kezirian GM. Laser in situ keratomileusis for myopia and astigmatism: 6 month results. J Cataract Refract Surg. 1998;24(6):758-64.

9. Holland SP, Srivannaboon S, Reinstein DZ. Avoiding serious corneal complications of laser assisted in situ keratomileusis and photorefractive keratectomy. Ophthalmology. 2000;107(4):640-52.

10. Seiler T, Holschbach A, Derse M, Jean B, Genth U. Complications of myopic photorefractive keratectomy with the excimer laser. Ophthalmology. 1994; 101(1):153-60

11. Pop M, Payette Y. Results of bilateral photorefractive keratectomy. Ophthalmology. 2000;107(3):472-9.

12. Lin RT, Maloney RK. Flap complications associated with lamellar refractive surgery. Am J Ophthalmol. 1999;127(2):129-36.

13. Webber SK, Lawless MA, Sutton GL, Rogers CM. Staphylococcal infection under a LASIK flap. Cornea. 1999;18(3):361-5.

14. Höfling-Lima AL, Farah ME, Montenegro L, Alvarenga LS, Chalita MRC, You MCZ. Alterações da microbiota conjuntival e palpebral após uso tópico de lomefloxacina e tobramicina na cirurgia de catarata e cirurgia refrativa. Arq Bras Oftalmol. 2002;65(1):21-9.

15. Chaib AR, Freitas D, Scarpi MJ, Guidugli T. Pesquisa laboratorial em endoftalmite. Arq Bras Oftalmol. 1997;60(3):250-7.

16. Schirmbeck T, Romão E, Rodrigues MLV, Figueiredo JFC. Endoftalmite: uma análise de 58 casos. Arq Bras Oftalmol. 2000;63(1):39-44.

17. Höfling-Lima AL, Moeller CTA, Yu MCZ, Branco BC, Farad ME. Microbiological efficacy of lomefloxacin and other drugs regarding microorganisms isolated from the human conjunctiva. Arq Bras Oftalmol. 2001;64(2):147-51.

18. Tengroth B, Epstein D, Fagerholm P, Hamberg-Nystrom H, Fitzsimmons TD. Excimer laser photorefractive keratectomy for myopia. Clinical results in sighted eyes. Ophthalmology. 1993;100(5):739-45.
19. Yee RW. Analgesic efficacy and safety of nonpreserved ketorolac tromethamine ophthalmic solution following radial keratotomy. Keratolac Radial Keratotomy Study Group. Am J Ophthalmol. 1998;125(4):472-80.

20. Arshinoff S, D'Addario D, Sadler C, Bilotta R, Johnson TM. Use of topical nonsteroidal anti-inflammatory drugs in excimer laser photorefractive keratectomy. J Cataract Refract Surg. 1994;20 Suppl:216-22.

21. Sher NA. Postoperative management of the PRK patient. Focal Points 1998; 10(1):6-10

22. Detorakis ET, Siganos DS, Houlakis VM, Kozobolis VP, Pallikaris IG. Microbiological examination of bandage soft contact lenses used in laser refractive surgery. J Refract Surg. 1998;14(6):631-5.

23. Chusid MJ, Davis SD. Polymorphonuclear leukocyte kinetics in experimentally induced keratitis. Arch Ophthalmol. 1985;103(2):270-4.

24. Chusid MJ, Shea ML. Quantitation of corneal inflammation by chemiluminescense. Arch Ophthalmol. 1986;104(10):1540-4.

25. Pavan-Langston D, Dunkel EC. Ocular varicella-zoster virus infection in the guinea pig. A new in vivo model. Arch Ophthalmol. 1989;107(7):1068-72.

26. Ohadi C, Litwin KL, Moreira H, Kwitko S, Gauderman WJ, Gritz AC, et al. Anti-inflammatory therapy and outcome in a guinea pig model of Pseudomonas keratitis. Cornea. 1992;11(5): 398-403.

27. Mori J, Ishizaki M, Senoo T. Effects of iodine on experimental allergic conjunctivitis. Nippon Ganka Gakkai Zasski. 2002;106(6):332-7. Japanese

28. Watanabe H, Sato S, Maeda N, Inoue Y, Shimomura Y, Tano Y. Bilateral corneal infection as a complication of laser in situ keratomileusis. Arch Ophthalmol. 1997;115(12):1593-4.

29. Moreira H. Tratamento da ceratite experimental por Pseudomonas aeruginosa com inibidores da cicloxigenase e lipoxigenase [tese]. São Paulo: Universidade Federal de São Paulo. Escola Paulista de Medicina; 1992.

30. Gritz DC, Lee TY, Kwitko S, McDonnell PJ. Topical anti-inflammatory agents in an animal model of microbial keratitis. Arch Ophthalmol. 1990;108(7):1001-5.

\section{CONGRESSO BRASILEIRO DE OFTALMOLOGIA}

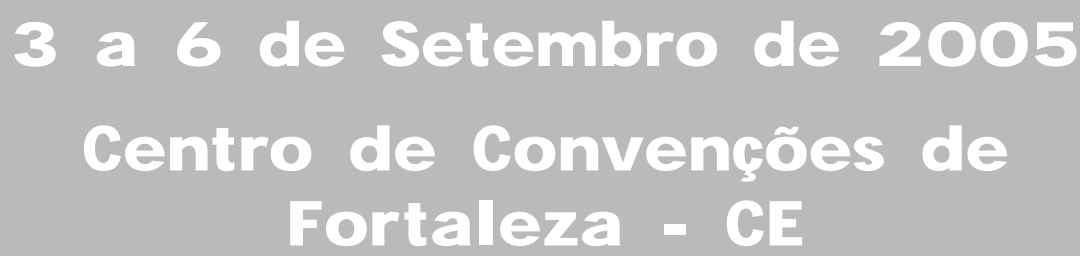

IN FORMAÇÕ ES: Secretaria Executiva: Arx Produções \& Eventos

Rua Júlio Siqueira, 970 - Dionísio Torres

60130-090 - Fortaleza (CE)

Fone: (85) 4011-1572 - Fax: 4011-1573

Email: cb02005@arxweb.com.br

Home-page: www.cbo2005.com.br 